\title{
Are blood lipids associated with microvascular complications among type 2 diabetes mellitus patients? A cross-sectional study in Shanghai, China
}

Hua Yang ${ }^{1}$, Doris Young ${ }^{2}$, Jian Gao ${ }^{3}$, Yuanzhi Yuan ${ }^{4}$, Minqian Shen ${ }^{4}$, Yuan Zhang ${ }^{4}$, Xueyan Duan ${ }^{5}$, Shanzhu Zhu ${ }^{1 *}$ (D) and Xiaoming Sun ${ }^{6 *}$

\begin{abstract}
Background: Although there are several studies to investigate the association between blood lipids and microvascular complications, these studies reported conflicting results. The aim of the current study was to explore the association between blood lipid parameters and the risk of microvascular complications, especially the doseresponse association between them, among community patients with type 2 diabetes mellitus (T2DM) in Shanghai, China.

Methods: The cross-sectional study was conducted in 6 community health service centers in Shanghai between December 2014 and December 2016.The associations between blood lipids and diabetic kidney disease (DKD) or diabetic retinopathy (DR) were assessed using multiple logistic regression. Restricted cubic spline (RCS) was employed to estimate the dose-response relation of blood lipids and the risk of microvascular complications.

Results: A total of 3698 participants were included in the final analysis to study the association between blood lipids and DKD, wherein 33.2\% of participants had DKD and 1374 were included for the analysis of the association between blood lipids and DR, wherein $23.2 \%$ of participants had DR. DKD odds ratio was increased by $1.16(95 \% \mathrm{Cl}, 1$. 08-1.25), 1.21(95\%Cl,1.13-1.30), 1.18(95\%Cl,1.10-1.26) for comparing fourth to first quartiles of triglycerides (TG), TG/ high-density lipoprotein cholesterol (HDL-C), non-HDL-C/HDL-C, respectively, and decreased by $0.83(95 \% \mathrm{Cl}, 0.78-0$. 89) for comparing fourth to first quartiles of HDL-C. Furthermore, the dose-response association between TG, HDL$\mathrm{C}, \mathrm{TG} / \mathrm{HDL}-\mathrm{C}$, non-HDL-C/HDL-C and the risk of DKD demonstrated turning points in TG of $1.90 \mathrm{mmol} / \mathrm{L}, \mathrm{HDL}-\mathrm{C}$ of 1 . $62 \mathrm{mmol} / \mathrm{L}, \mathrm{TG} / \mathrm{HDL}-\mathrm{C}$ of 2.00 , non-HDL-C/HDL-C of 3.09, respectively. However, no significant association was found between blood lipid parameters and DR.
\end{abstract}

Conclusions: This community-based study indicated that TG, HDL-C, TG/HDL-C, non-HDL-C/HDL-C were independently associated with DKD but not DR.

Keywords: Blood lipids, Diabetic kidney disease, Diabetic retinopathy, Type 2 diabetes mellitus

\footnotetext{
*Correspondence: shanzhu_zhu@163.com; xm_sun2003@aliyun.com

${ }^{1}$ Department of General Practice, Zhongshan Hospital of Fudan University,

Shanghai, China

${ }^{6}$ Pudong Institute for Health Development, Shanghai, China

Full list of author information is available at the end of the article
}

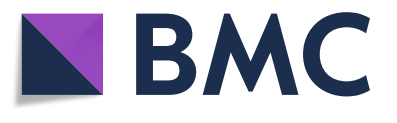

(c) The Author(s). 2019 Open Access This article is distributed under the terms of the Creative Commons Attribution 4.0 International License (http://creativecommons.org/licenses/by/4.0/), which permits unrestricted use, distribution, and reproduction in any medium, provided you give appropriate credit to the original author(s) and the source, provide a link to the Creative Commons license, and indicate if changes were made. The Creative Commons Public Domain Dedication waiver (http://creativecommons.org/publicdomain/zero/1.0/) applies to the data made available in this article, unless otherwise stated. 


\section{Background}

Diabetic kidney disease (DKD) and diabetic retinopathy (DR) are major microvascular complications of type 2 diabetes mellitus (T2DM), which produces an enormous burden to national health-care systems. It has been estimated that 20 to $40 \%$ of patients with diabetes mellitus will develop DKD [1], which is the leading cause of end-stage renal disease in the Western countries [2] and ranks second in China [3], Over one-third of patients with diabetes mellitus have signs of DR [4], which is the leading cause of blindness in developed countries [5].

Several studies have demonstrated that hyperglycemia and hypertension play important roles in the development and progression of DKD and DR $[6,7]$. However, other studies demonstrated that in spite of the reaching of recommended blood glucose and blood pressure targets, the risk for DKD and DR still remained high among T2DM patients [8-10]. Thus, exploring the association between microvascular complications and other metabolic elements has become increasingly important. More and more evidences suggest the involvement of dyslipidemia in the development of DKD and DR [11, 12]. Some studies have demonstrated that plasma triglycerides (TG), high-density lipoprotein cholesterol (HDL-C), and TG/HDL-C ratio, but not low-density lipoprotein cholesterol (LDL-C) were associated with microvascular complications [13-15]. Few other studies reported conflicting results. The Kidney Early Evaluation Program (KEEP) [16] demonstrated that only HDL-C was associated with urinary albumin to creatinine ratio (UACR). Sjølie AK et al. [17] found that TG, but not cholesterol was a significant risk factor for moderate-severe non-proliferative retinopathy and proliferative retinopathy. However, the association between blood lipids and microvascular complications still were poorly defined in Chinese community patients with T2DM.

Therefore, the objective of the present cross-sectional study was to explore the association, especially the dose-response association, between blood lipid parameters and the risk of microvascular complications among the target populations.

\section{Methods}

A cross-sectional study was conducted among T2DM patients in six communities of Shanghai. The study protocol was approved by the Ethics Committee of Zhongshan Hospital of Fudan University (B2016-029).

\section{Population}

In the current study, a stratified random sampling procedure was conducted to recruit participants from December 2014 to December 2016. Three districts were purposefully selected from sixteen districts of Shanghai, where Xuhui represented old central district, Pudong the new central and Jiading the outer fringe district, respectively. Next, we selected two community health centers ( $\mathrm{CHCs}$ ) randomly from each district, with the inclusion of six CHCs in total. Finally, we randomly recruited participants who were 18 years of age or older, diagnosed with T2DM for $\geq 3$ months previously, incorporated into diabetes mellitus management system of China [18] from these six CHCs. Patients with pregnancy were not included in the current study. Considering the prevalence of microvascular complications in type 2 diabetes patients in previous studies [1, 4], and with a questionnaire failure rate of $20 \%$, we required a minimum sample size of 1920 to be studied. A total of 3977 subjects were finally enrolled. Written informed consent was obtained from all the volunteers.

\section{Measurements}

All data were obtained from field investigations, including a face-to-face interview, physical examination and biochemical measurement. The interview questionnaire contained age, gender, educational attainment, marital status, smoking status, family history of diabetes, duration of diabetes, current medical treatment, history of hypertension, coronary heart disease and stroke. Physical examination included the measurements of body weight, height, and blood pressure. Body weight and height were measured by trained investigators using standardized weight scales and meter measures. Blood pressure was measured with an arm type electronic sphygmomanometer or a manual one three times after resting for more than $10 \mathrm{~min}$ and then the average was taken. Biochemical measurement, including fasting blood glucose (FBG), hemoglobin A1c (HbA1c), total cholesterol (TC), TG, LDL-C, HDL-C, blood urea nitrogen (BUN), creatinine (CRE), uric acid (UA), UACR and urine analysis, were performed under a fasting condition of participants in the morning. Non-mydriatic fundus photography was obtained by using standard vision digital color fundus camera (Smartscope PRO from Optomed Oy, Oulu, Finland, or Canon CR-2 from Canon Inc.) by trained general practitioners (GPs). At least two-field retinal photographs based on macula fovea as the center were taken according to the standard protocol.

\section{Case definitions}

Albuminuria was defined as $\mathrm{UACR} \geq 30 \mathrm{mg} / \mathrm{g}$ in a single random urine sample, and those whose urine analysis showed microscopic hematuria or microscopic leucocyturia were excluded. DKD was defined as either albuminuria or an estimated glomerular filtration rate (eGFR) of < $60\left(\mathrm{ml} \cdot \mathrm{min}^{-1}\left(173 \mathrm{~m}^{2}\right)^{-1}\right)$ according to the Modification of Diet in Renal Disease [19] and the formula was as follows: $\operatorname{eGFR}\left(\mathrm{ml} \cdot \mathrm{min}^{-1} \cdot\left(173 \mathrm{~m}^{2}\right)^{-1}\right)=186 \times \mathrm{CRE}(\mathrm{mg} / \mathrm{dl})^{-1.154} \times$ $\operatorname{age}^{-0.203}(\times 0.742$,if female). Those with albuminuria or estimated glomerular filtration rate $(\mathrm{eGFR})<60\left(\mathrm{ml} \cdot \mathrm{min}^{-1}\right.$. $\left.\left(173 \mathrm{~m}^{2}\right)^{-1}\right)$ due to non-diabetic kidney disease (NDKD), 
determined by the confirmed medical history and records of NDKD by physicians were excluded.

DR was defined as having medical history of laser treatment for DR or being diagnosed with DR according to the International Clinical Grading Standards of Diabetic Retinopathy (2002) by ophthalmologists based on non-mydriatic fundus photography. The unqualified fundus photographs according to the standard protocol were deleted. The results were categorized into two levels: those with DR and without DR.

Hypertension was defined as either systolic blood pressure (SBP) $\geq 140 \mathrm{mmHg}$ or diastolic blood pressure (DBP) $\geq 90 \mathrm{mmHg}$. Participants who received antihypertensive treatment were also included in the hypertension definition.

\section{Quality control}

The investigators including GPs, public health physicians and nurses were trained before survey. The training was performed by two GPs of Department of General Practice of Zhongshan Hospital, Fudan University. The training courses involved the skills of interview, how to fill in the questionnaire and measure body weight, height, and blood pressure, and how to guide the participants to collect urine specimens. Quality control visits by the study team were conducted at each community to minimize the missing data.

\section{Statistical analysis}

Statistical analysis was carried out using SPSS software, version 17.0 (SPSS Inc., Chicago USA), SAS software, version 9.2 (SAS Institute, Cary, NC), and $\mathrm{R}$, version 3.3.0 (http://www.r-project.org).

Data were presented as percentages or means \pm standard deviations. Variables were examined between groups using Chi-square tests for the percentages and unpaired $\mathrm{t}$ tests for the mean values. The parameters of blood lipids, including TC, TG, LDL-C, HDL-C, Non-HDL-C, TG/HDL-C, and Non-HDL-C/HDL-C were divided into quartiles according to the linear scores. The associations between blood lipids and DKD or DR were assessed via multiple logistic regression. Multiple logistic regression models were fitted with or without DKD or DR as dependent variables and the quartiles of the blood lipid parameters as independent variables. The lowest quartile of the blood lipid parameters were used as reference in this model. Adjustments were made for potential confounders, including age, gender, smoking status, family history of diabetes, duration of diabetes and current medical treatment, comorbid hypertension, comorbid CAD, comorbid stroke, BMI, HbA1c level, and SBP. Furthermore, restricted cubic spline (RCS) was employed to estimate the dose-response relationship of blood lipids with the risk of microvascular complications [20]. Odds ratios (ORs) and 95\% confidence intervals (CIs) were calculated. A two-tailed alpha with $P<0.05$ was considered statistically significant for all analyses.

\section{Results \\ Characteristics of participants}

A total of 3977 participants without missing basic socio-demographic characteristics data were enrolled in this study. Of these, 3698 participants were included in the current analysis to study the association between blood lipids and DKD, and 1374 participants were included in the current analysis to study the association between blood lipids and DR (Fig. 1).

Analysis of kidney disease revealed that $33.2 \%$ of participants had DKD. Participants with DKD had older age, longer duration of diabetes, higher proportion of using insulin, having comorbid hypertension, CAD, stroke, DR, higher levels of BMI, SBP, HbA1c, TG, HDL-C, non-HDL-C, and lower level of eGFR than those without DKD $(P<0.05$, Table 1$)$.

In the retinopathy disease analysis, there were $23.2 \%$ of participants with DR. Participants with DR had younger age, longer duration of diabetes, higher proportion of smoking, using oral medicine+insulin, having positive UACR, higher levels of SBP, HbA1c than those without DR $(P<0.05$, Table 1$)$.

\section{Association between blood lipids and microvascular complications}

T2DM participants with higher levels of TG, TG/ HDL-C, non-HDL-C/HDL-C were more likely to suffer from DKD. The OR for the participants with DKD comparing fourth to first quartiles of TG, TG/HDL-C, non-HDL-C/HDL-C were 1.16(95\%CI,1.08-1.25), $1.21(95 \% \mathrm{CI}, 1.13-1.30)$, and $1.18(95 \% \mathrm{CI}, 1.10-1.26)$, respectively. T2DM participants with higher HDL-C levels were less likely to suffer from DKD. The OR for the participants with DKD comparing fourth to first quartiles of HDL-C was 0.83 (95\%CI, 0.77-0.89). The trends were statistically significant for associations between $\mathrm{TG}$, HDL-C, TG/HDL-C, non-HDL-C/HDL-C and the risk of DKD ( $P$ for trend<0.001) (Fig. 2). This was performed after adjusting for age, gender, smoking status, family history of diabetes, duration of diabetes, current medical treatment, comorbid hypertension, CAD, stroke, BMI, HbA1c level, and SBP.

Furthermore, a dose-response association between TG, HDL-C, TG/HDL-C, non-HDL-C/HDL-C and the risk of DKD was found by spline analysis. When TG was lower than $1.90 \mathrm{mmol} / \mathrm{L}$, the possibility of having DKD showed a significant increase along with increased TG levels (OR $=1.48,95 \% \mathrm{CI}, 1.21-1.81, P<0.001)$. When TG/HDL-C was lower than 2.00, the possibility of having DKD showed a significant increase along with increased TG/ 


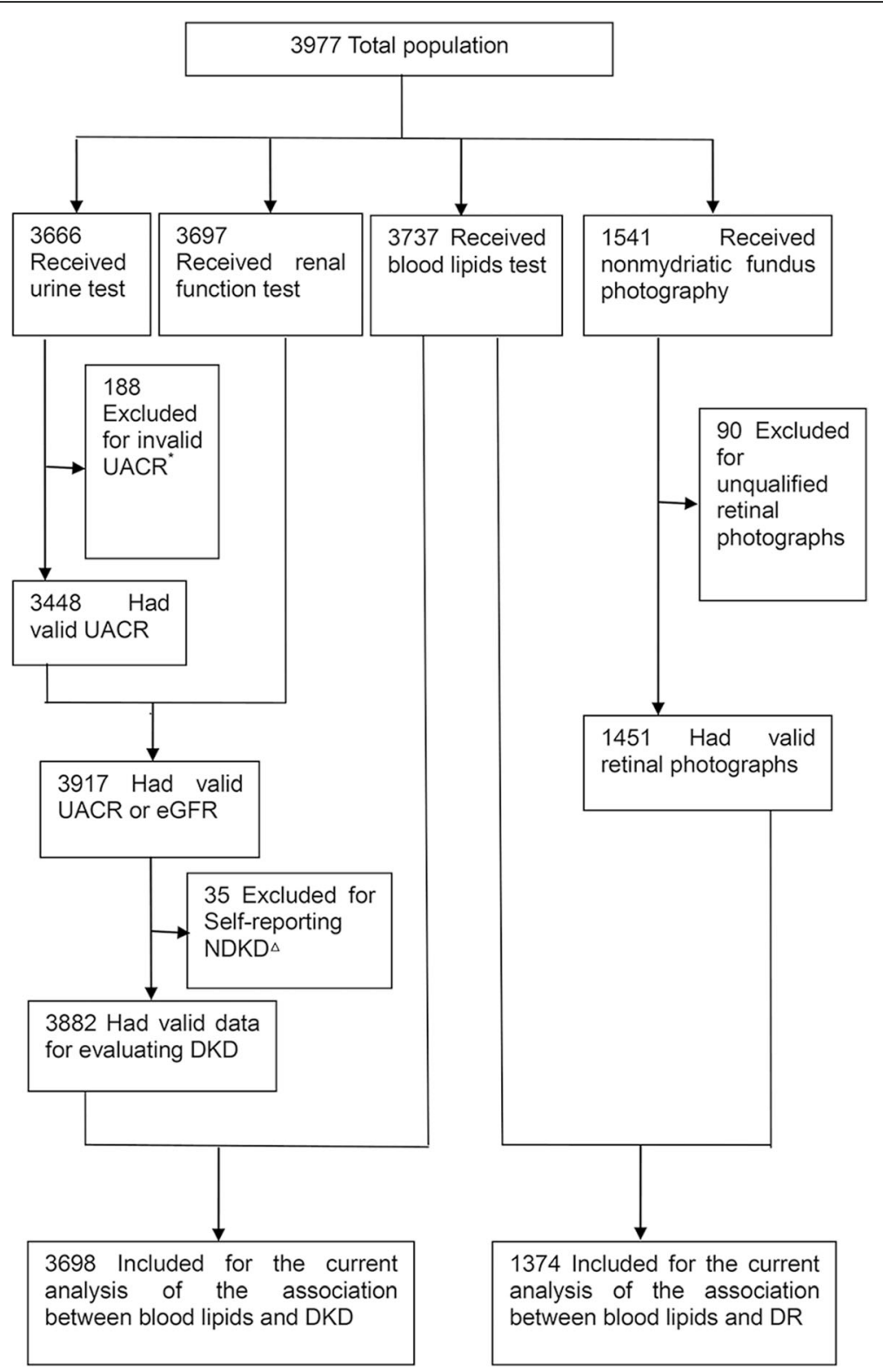

Fig. 1 Inclusion/exclusion criteria of the study participants for assessing the association between blood lipids and microvascular complications. *: Invalid UACR was defined as having microscopic hematuria or microscopic leucocyturia in urine routine. ${ }^{\star}$ : Nondiabetic Kidney Disease (NDKD) was determined by physicians according to medical history and records of the participant

HDL-C levels (OR = 1.52, 95\%CI, 1.30-1.78, $P<0.001)$. When non-HDL-C/HDL-C was lower than 3.09, 3.07 in men and 2.63 in women, respectively, the possibility of having DKD showed a significant increase along with increased Non-HDL-C/HDL-C levels (OR $=1.34$, 95\%CI,1.17-1.53, $P<0.001)$. When HDL-C was lower than $1.62 \mathrm{mmol} / \mathrm{L}$, the possibility of having DKD showed a significant decrease along with the increase of HDL-C levels (OR $=0.42,95 \% \mathrm{CI}, 0.31-0.58, P<$ 0.001) (Table 2, Fig. 3). Saturation effect was found when the levels of TG, HDL-C, TG/HDL-C, and non-HDL-C/HDL-C were higher than each turning point (Fig. 3). There was no interaction between gender and blood lipids effect on the risk of DKD.

In the current analysis, no significant association was found between blood lipid parameters and DR (Fig. 4).

\section{Discussion}

In this current study, higher levels of TG, TG/ HDL-C, non-HDL-C/HDL-C and lower levels of HDL-C, were associated with DKD, but not with DR. Notably, such associations were independent of several confounding factors, such as age, gender, smoking status, family history of diabetes, duration of 
Table 1 Socio-demographic and clinical characteristics of participants according to DKD and DR

\begin{tabular}{|c|c|c|c|c|c|c|}
\hline \multirow[t]{2}{*}{ Variable } & \multicolumn{3}{|l|}{ DKD } & \multicolumn{3}{|l|}{$\mathrm{DR}$} \\
\hline & No $\left(n^{d}=2472\right)$ & Yes $\left(n^{d}=1226\right)$ & $p^{e}$ & No $\left(n^{d}=1055\right)$ & Yes $\left(n^{d}=319\right)$ & $P^{f}$ \\
\hline Gender (male), n (\%) & 1086(43.9) & $518(42.3)$ & $0.332^{h}$ & $442(41.9)$ & $142(44.5)$ & $0.407^{h}$ \\
\hline Age (years), mean $\pm S D$ & $66.8 \pm 8.1$ & $69.1 \pm 8.7$ & $<0.001^{\mathrm{g}}$ & $65.7 \pm 7.4$ & $64.8 \pm 7.6$ & $0.045^{\mathrm{g}}$ \\
\hline Current smoker, n (\%) & $443(17.9)$ & $172(14.0)$ & $0.003^{h}$ & 162(15.4) & $66(20.7)$ & $0.025^{h}$ \\
\hline Family history of diabetes mellitus, $\mathrm{n}(\%)$ & 703(29.8) & $345(29.7)$ & $0.934^{h}$ & $372(37.2)$ & 119(38.6) & $0.649^{h}$ \\
\hline Duration of diabetes mellitus(years), mean \pm SD & $8.8 \pm 6.1$ & $9.8 \pm 6.6$ & $<0.001^{\mathrm{g}}$ & $8.1 \pm 5.7$ & $11.2 \pm 6.6$ & $<0.001^{9}$ \\
\hline \multicolumn{7}{|l|}{ Current medical treatment, n (\%) } \\
\hline Without medicine & 209(9.1) & $96(8.4)$ & $<0.001^{h}$ & $50(5.4)$ & $7(2.4)$ & $<0.001^{h}$ \\
\hline Oral medicine & 1769(77.1) & $847(73.7)$ & & 775(83.0) & 210(71.2) & \\
\hline Insulin & 134(5.8) & 113(9.8) & & $45(4.8)$ & $23(7.8)$ & \\
\hline Oral medicine + insulin & 181(7.9) & $93(8.1)$ & & $64(6.9)$ & $55(18.6)$ & \\
\hline Hypertension, n (\%) & 1789(72.4) & $1012(82.5)$ & $<0.001^{h}$ & 795(75.4) & $245(76.8)$ & $0.598^{h}$ \\
\hline$C A D, n(\%)$ & 253(10.2) & $155(12.6)$ & $0.028^{h}$ & $98(9.3)$ & $39(12.2)$ & $0.125^{h}$ \\
\hline Stroke, n (\%) & 189(7.6) & $122(10.0)$ & $0.017^{h}$ & $98(9.3)$ & $32(10.0)$ & $0.691^{h}$ \\
\hline BMI $\left(\mathrm{kg} / \mathrm{m}^{2}\right)$, mean \pm SD & $25.2 \pm 3.2$ & $25.7 \pm 3.5$ & $<0.001^{\mathrm{g}}$ & $25.3 \pm 3.2$ & $25.5 \pm 3.2$ & $0.460^{g}$ \\
\hline $\mathrm{SBP}(\mathrm{mmHg})$, mean $\pm \mathrm{SD}$ & $134.4 \pm 15.1$ & $139.0 \pm 18.0$ & $<0.001^{\mathrm{g}}$ & $137.1 \pm 17.3$ & $140.4 \pm 19.0$ & $0.004^{g}$ \\
\hline $\mathrm{DBP}(\mathrm{mmHg})$, mean $\pm \mathrm{SD}$ & $79.0 \pm 8.3$ & $79.1 \pm 8.7$ & $0.720^{g}$ & $79.1 \pm 8.7$ & $78.3 \pm 9.1$ & $0.189^{g}$ \\
\hline $\mathrm{HbA1c}(\%)$, mean $\pm \mathrm{SD}$ & $7.0 \pm 1.3$ & $7.5 \pm 1.7$ & $<0.001^{9}$ & $7.0 \pm 1.2$ & $7.6 \pm 1.5$ & $<0.001^{9}$ \\
\hline $\mathrm{TC}(\mathrm{mmol} / \mathrm{L})$, mean $\pm \mathrm{SD}$ & $4.7 \pm 1.0$ & $4.8 \pm 1.0$ & $0.266^{9}$ & $4.5 \pm 1.0$ & $4.4 \pm 1.2$ & $0.164^{\mathrm{g}}$ \\
\hline $\mathrm{TG}(\mathrm{mmol} / \mathrm{L})$, mean $\pm \mathrm{SD}$ & $1.6 \pm 1.1$ & $1.7 \pm 1.1$ & $<0.001^{\mathrm{g}}$ & $1.6 \pm 1.1$ & $1.5 \pm 0.9$ & $0.193^{g}$ \\
\hline $\mathrm{LDL}-\mathrm{C}(\mathrm{mmol} / \mathrm{L})$, mean $\pm \mathrm{SD}$ & $2.6 \pm 0.9$ & $2.6 \pm 1.0$ & $0.613^{\mathrm{g}}$ & $2.7 \pm 0.8$ & $2.6 \pm 0.9$ & $0.153^{g}$ \\
\hline $\mathrm{HDL}-\mathrm{C}(\mathrm{mmol} / \mathrm{L})$, mean $\pm \mathrm{SD}$ & $1.4 \pm 0.4$ & $1.3 \pm 0.4$ & $<0.001^{\mathrm{g}}$ & $1.4 \pm 0.4$ & $1.3 \pm 0.4$ & $0.217^{g}$ \\
\hline $\mathrm{TG} / \mathrm{HDL}-\mathrm{C}$, mean $\pm \mathrm{SD}$ & $1.3 \pm 1.2$ & $1.4 \pm 1.2$ & $<0.001^{\mathrm{g}}$ & $1.3 \pm 1.1$ & $1.2 \pm 1.0$ & $0.366^{9}$ \\
\hline Non-HDL-C (mmol/L), mean \pm SD & $3.3 \pm 1.0$ & $3.4 \pm 1.0$ & $0.002^{g}$ & $3.2 \pm 1.0$ & $3.1 \pm 1.1$ & $0.294^{9}$ \\
\hline Non-HDL-C/ HDL-C, mean \pm SD & $2.6 \pm 1.1$ & $2.8 \pm 1.1$ & $<0.001^{\mathrm{g}}$ & $2.5 \pm 1.0$ & $2.5 \pm 1.0$ & $0.883^{g}$ \\
\hline eGFR ${ }^{a}\left(\mathrm{ml} \cdot \min ^{-1} \cdot\left(173 \mathrm{~m}^{2}\right)^{-1}\right)$, mean $\pm \mathrm{SD}$ & $102.4 \pm 26.3$ & $90.0 \pm 32.2$ & $<0.001^{\mathrm{g}}$ & $102.5 \pm 30.1$ & $105.4 \pm 31.2$ & $0.147^{g}$ \\
\hline \multicolumn{7}{|l|}{$\mathrm{UACR}^{\mathrm{b}}(\mathrm{mg} / \mathrm{g}), \mathrm{n}(\%)$} \\
\hline$<30$ & 2092(100) & 108(9.0) & $<0.001^{h}$ & $688(69.0)$ & $181(59.9)$ & $0.010^{h}$ \\
\hline $30-300$ & $0(0)$ & $967(80.7)$ & & $274(27.5)$ & 104(34.4) & \\
\hline$>300$ & $0(0)$ & 123(10.3) & & $35(3.5)$ & 17(5.6) & \\
\hline $\mathrm{DR}^{\mathbf{c}}, \mathrm{n}(\%)$ & $190(20.8)^{d}$ & $125(28.2)$ & $0.003^{h}$ & - & - & - \\
\hline
\end{tabular}

$C A D$ coronary heart disease, $B M I$ body mass index, eGFR estimated glomerular filtration rate, UACR urinary albumin creatinine ratio, $D R$ diabetic retinopathy ${ }^{a}$ :eGFR was calculated using the formula of Modification of Diet in Renal Disease formula: eGFR $\left(\mathrm{ml}^{-} \cdot \mathrm{min}^{-1} \cdot\left(1.73 \mathrm{~m}^{2}\right)^{-1}\right)=186 \times C R E(\mathrm{mg} / \mathrm{dl})^{-1.154}$ $\times$ age ${ }^{-0.203}(\times 0.742$, if female)

b: UACR was measured on a single random urine sample and was calculated from urinary albumin creatinine ratio

c: DR was defined based on the International Clinical Grading Standards of Diabetic Retinopathy (2002) by ophthalmologist according to the retinal photographs. The results were categorized into two levels: with DR or without DR

${ }^{\mathrm{d}}$ : Missing values weren't imputed into the study database or case-wise deleted; therefore, the number of respondents included in analysis differed by variable of interest

e: Participants with DKD in comparison to those without DKD

f:Participants with DR in comparison to those without DR

${ }^{9}$ :using $t$ test

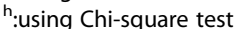

diabetes, current medical treatment, hypertension, CAD, stroke, BMI, HbA1c level, and SBP. Furthermore, the dose-response association between TG, HDL-C, TG/HDL-C, non-HDL-C/HDL-C and the risk of DKD were found by RCS, which demonstrated the turning points in TG of $1.90 \mathrm{mmol} / \mathrm{L}, \mathrm{HDL}-\mathrm{C}$ of 1.62 $\mathrm{mmol} / \mathrm{L}$, TG/HDL-C of 2.00 , and non-HDL-C/HDL-C of 3.09, respectively.

Stadler K et al. study reported the changes in serum lipid levels in T2DM and demonstrated that different blood lipids had different variation trends [21]. Most of the previous studies on the association of DKD and 


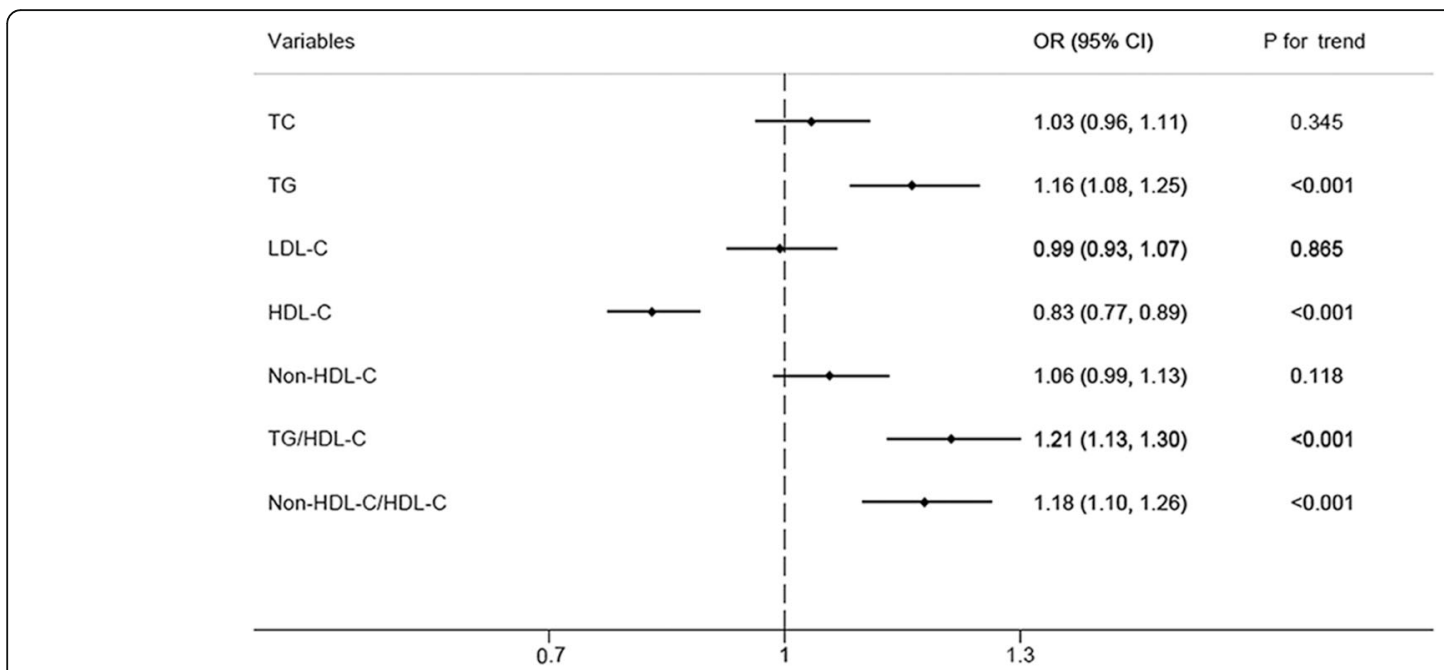

Fig. 2 Odds ratio (OR) for participants with diabetic kidney disease, comparing fourth to first quartiles of TC, TG, LDL-C, HDL-C, Non-HDL-C, TG/ HDL-C, or Non-HDL-C/HDL-C. This was performed after adjusting for age, sex, family history of diabetes mellitus, duration of diabetes mellitus, current medical treatment, smoke status, comorbid hypertension, CAD, stroke, BMI, HbA1c, SBP

blood lipids demonstrated that high TG was associated with DKD [22-25], although several other studies showed no association [26, 27]. Zoppini G et al. [28] in their study followed 1987 T2DM outpatients with normal or near-normal kidney function at baseline for 5 years for the occurrence of chronic kidney disease(CKD). (CKD) They found that higher plasma levels of HDL-C were associated with lower risk of incidence of CKD in adult patients with T2DM. A large observational retrospective study of diabetic population conducted in Italy [29] demonstrated that TG $\geq 150 \mathrm{mg} / \mathrm{dL}$ increased the risk of low eGFR by $26 \%$, albuminuria by $19 \%$, whereas $\mathrm{HDL}-\mathrm{C}<40$ $\mathrm{mg} / \mathrm{dL}$ in men and $<50 \mathrm{mg} / \mathrm{dL}$ in women increased the risk of low eGFR by $27 \%$, and albuminuria by $24 \%$. Chang et al. [30] reported that the development of albuminuria was associated with higher TG and lower HDL-C levels, but not with higher LDL-C level in T2DM patients. Similar results were obtained in our current study. In addition, interesting findings of the current study include higher lipid ratios, where both TG/HDL-C and non-HDL-C/ HDL-C were significantly associated with the increased possibility of having DKD. With respect to single lipid parameter usage, lipid ratios may highlight the opposite contribution of single parameters on DKD [15]. Kyung-Jin Yun et al. [31] reported that TG/HDL-C ratio may affect the development and progression of DKD in patients with T2DM and advanced DR. Zoppini G et al. [15] also found that the TG/HDL-C ratio was associated with an increased

Table 2 Dose-response association between blood lipids and the risk of DKD

\begin{tabular}{|c|c|c|c|c|c|c|c|c|}
\hline \multirow[t]{2}{*}{ Exposure Variables } & \multicolumn{2}{|l|}{ TG } & \multicolumn{2}{|l|}{$\mathrm{HDL}-\mathrm{C}$} & \multicolumn{2}{|l|}{$\mathrm{TG} / \mathrm{HDL}-\mathrm{C}$} & \multicolumn{2}{|c|}{ Non-HDL-C/HDL-C } \\
\hline & OR $(95 \% \mathrm{Cl})$ & $P$ & OR $(95 \% \mathrm{Cl})$ & $P$ & OR (95\%Cl) & $P$ & OR (95\%Cl) & $P$ \\
\hline \multicolumn{9}{|l|}{ Total } \\
\hline Turning point & $1.90 \mathrm{mmol} / \mathrm{L}$ & & $1.62 \mathrm{mmol} / \mathrm{L}$ & & 2.00 & & 3.09 & \\
\hline Lower than turning point & $1.48(1.21-1.81)$ & $<0.001$ & $0.42(0.31-0.58)$ & $<0.001$ & $1.52(1.30-1.78)$ & $<0.001$ & $1.34(1.17-1.53)$ & $<0.001$ \\
\hline Higher than turning point & $0.99(0.89-1.09)$ & 0.793 & $1.68(0.95-2.95)$ & 0.074 & $0.95(0.87-1.05)$ & 0.316 & $0.97(0.85-1.10)$ & 0.603 \\
\hline \multicolumn{9}{|l|}{ Men } \\
\hline Turning point & - & & $1.62 \mathrm{mmol} / \mathrm{L}$ & & 1.96 & & 3.07 & \\
\hline Lower than turning point & - & - & $0.43(0.27-0.70)$ & 0.001 & $1.51(1.18-1.95)$ & 0.001 & $1.40(1.13-1.73)$ & 0.002 \\
\hline Higher than turning point & - & - & $1.15(0.41-3.21)$ & 0.789 & $0.98(0.87-1.10)$ & 0.715 & $1.01(0.84-1.22)$ & 0.932 \\
\hline \multicolumn{9}{|l|}{ Women } \\
\hline Turning point & - & & $1.61 \mathrm{mmol} / \mathrm{L}$ & & 2.09 & & 2.63 & \\
\hline Lower than turning point & - & - & $0.43(0.28-0.68)$ & $<0.001$ & $1.59(1.29-1.94)$ & $<0.001$ & $1.45(1.14-1.85)$ & 0.003 \\
\hline Higher than turning point & - & - & $1.72(0.87-3.39)$ & 0.120 & $0.88(0.76-1.03)$ & 0.119 & $0.97(0.84-1.11)$ & 0.620 \\
\hline
\end{tabular}

Adjusted for age, sex, family history of diabetes mellitus, duration of diabetes mellitus, current medical treatment, smoke status, comorbid hypertension, comorbid CAD, comorbid stroke, BMI, HbA1c, SBP 


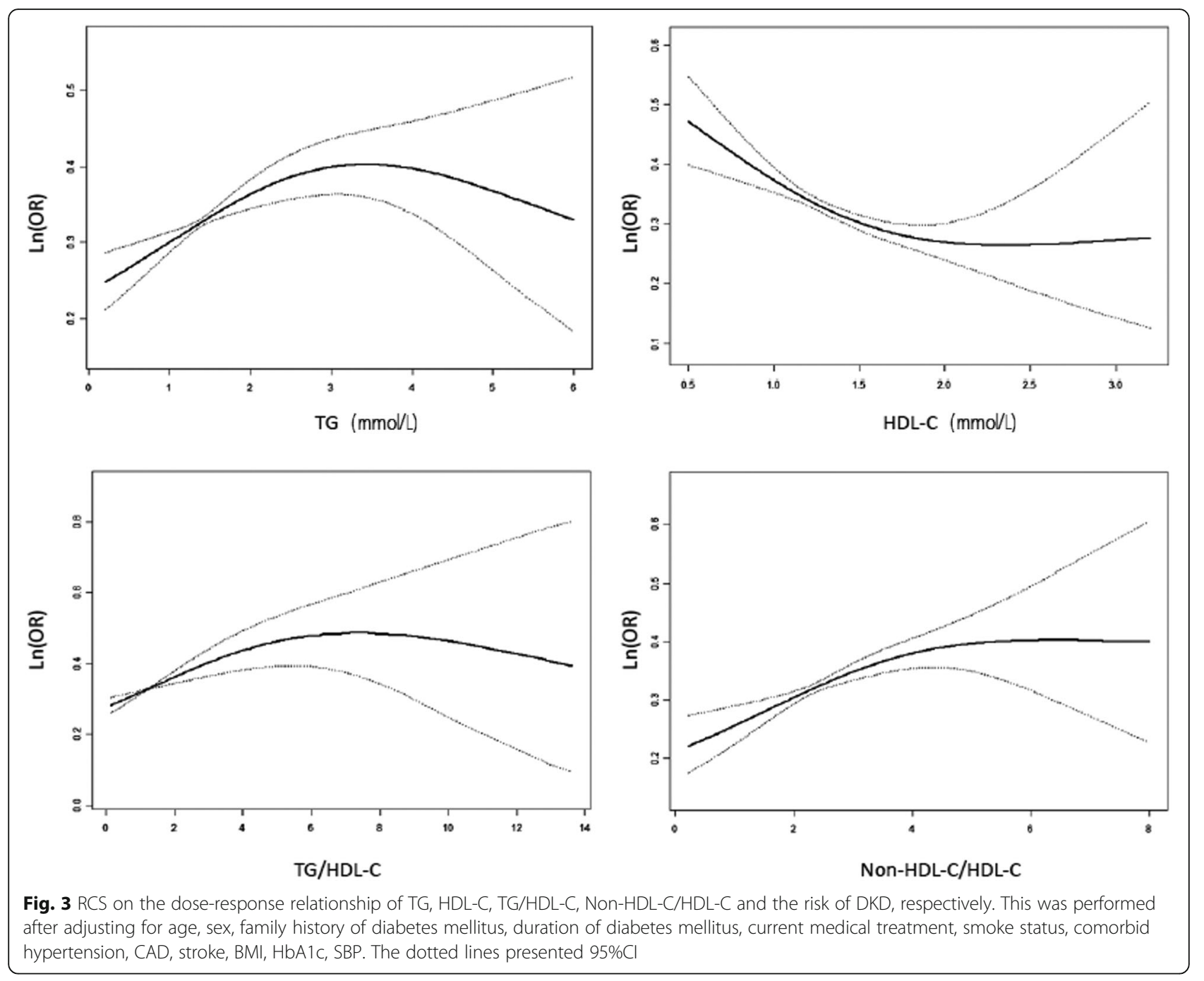

incidence of CKD in T2DM patients without prior cardiovascular disease after follow-up for 4.9 years. With regard to non-HDL-C/HDL-C ratio, some studies [32-34] showed that the ratio was probably a better predictor of metabolic syndrome, insulin resistance, arterial stiffness, and coronary heart disease. However, the relation of non-HDL-C/HDL-C ratio with DKD was not clearly presented in the previous studies. Furthermore, the dose-response association was found between TG, HDL-C, TG/HDL-C, non-HDL-C/ HDL-C and the risk of DKD in the current study. When TG, TG/HDL-C, HDL-C, non-HDL-C/HDL-C were lower than each turning point, the possibility of having DKD showed significant changes along with the variations of each blood lipid levels. These findings would caution us to pay more attention to mild abnormal blood lipids levels.

Previous studies on the association between DR and blood lipids showed controversial results. Some studies $[15,35,36]$ reported positive association between blood lipid parameters and DR, while others [13,37] showed negative associations. Our study was concordant with the latter results. We showed a positive association between dyslipidemia and DKD, but negative with DR, which may be explained by different mechanisms of action of blood lipids on these two complications. Diabetic microvascular disease has complex pathogenesis, which involves endothelial dysfunction, chronic low-grade inflammation, advanced end-glycation products, oxidative stress, and abnormalities in cytokines, fibrinolysis and coagulation $[38,39]$. Although the two types of microvascular diseases, DKD and DR were found to co-exist in the same patient, they were associated with different patho- physiologies.

According to the current study findings, high attention should be paid to TG, HDL-C, TG/HDL-C, and non-HDL-C/HDL-C levels in the management of DKD, especially in Chinese T2DM patients. A systematic review by Jing [40] found that the incidence of vascular complications and mortality were different in Western and Asian T2DM patients. Western patients demonstrated higher cardiovascular death rates and major coronary events, 


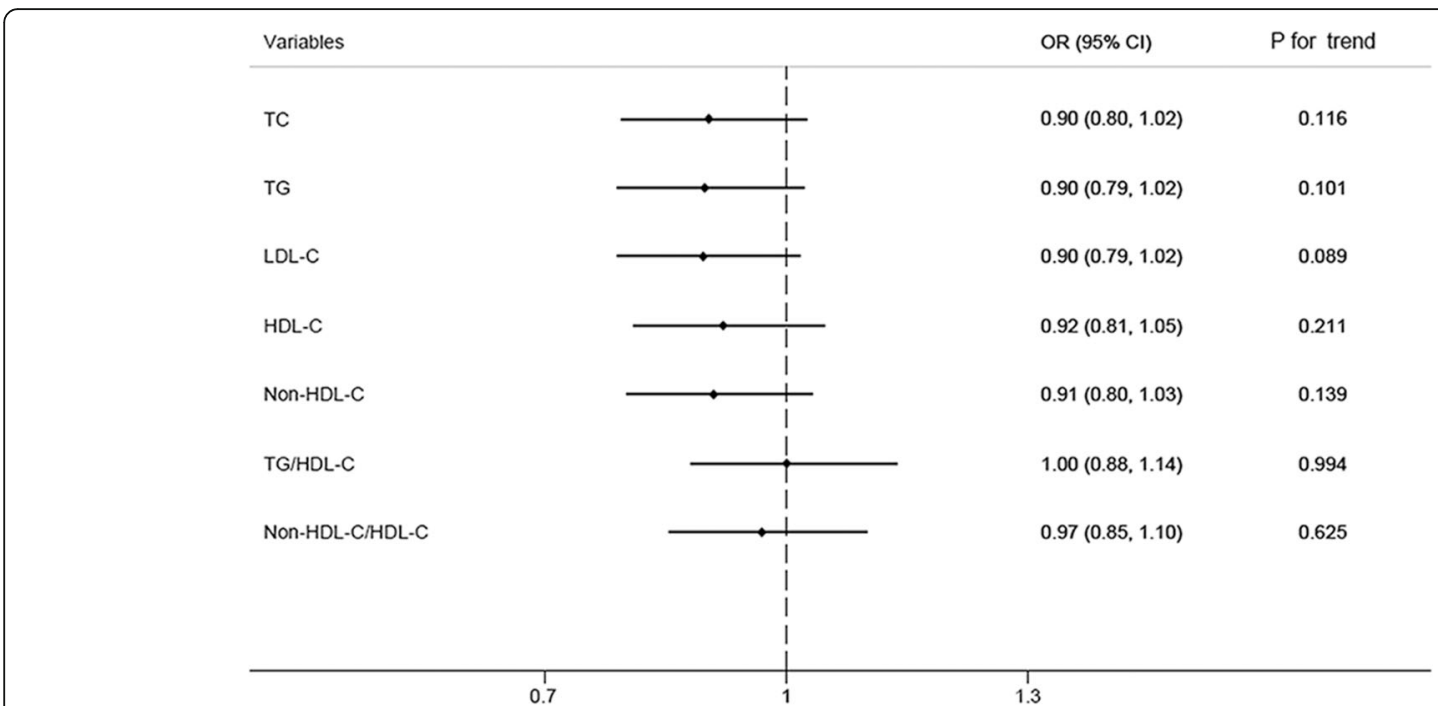

Fig. 4 Odds ratio (OR) for participants with diabetic retinopathy comparing fourth to first quartiles of TC, TG, LDL-C, HDL-C, Non-HDL-C, TG/HDLC, or Non-HDL-C/HDL-C. This was done by adjusting for age, sex, family history of diabetes mellitus, duration of diabetes mellitus, current medical treatment, smoke status, comorbid hypertension, CAD, stroke, BMI, HbA1c, SBP

whereas Asian patients showed higher incidence of major cerebrovascular and microvascular events, including nephropathy and retinopathy. Integrated lipid targets other than LDL-C should be established to prevent and control the microvascular complications, especially DKD, in the diabetic patients.

However, our study has some limitations. (1)The blood and urine measurements were conducted by each $\mathrm{CHC}$ separately, but they all met the qualified standards. (2)The response rate of receiving non-mydriatic fundus photography was low, which may generate selection bias. (3)The study involved a cross-sectional design, and therefore, only association rather than causation could be evaluated. Longitudinal studies would be helpful to further explain the relationship between blood lipids and microvascular complications among T2DM patients.

\section{Conclusions}

In conclusion, this community-based study indicated that TG, HDL-C, TG/HDL-C, non-HDL-C/HDL-C were independently associated with DKD but not with DR. The turning points in TG, HDL-C, TG/HDL-C, non-HDL-C/HDL-C associated with DKD were also found. These findings may be helpful in tailoring the screening and therapeutic strategies on microvascular complications in T2DM patients.

\section{Abbreviations}

BUN: blood urea nitrogen; CHCs: community health centers; Cls: confidence intervals; CKD: chronic kidney disease; CRE: creatinine; DBP: diastolic blood pressure; DKD: diabetic kidney disease; DR: diabetic retinopathy; eGFR: estimated glomerular filtration rate; FBG: fasting blood glucose; GPs: general practitioners; $\mathrm{HbA1c}$ : hemoglobin A1c; HDL-C: high-density lipoprotein cholesterol; KEEP: Kidney Early Evaluation Program; LDL-C: lowdensity lipoprotein cholesterol; NDKD: non-diabetic kidney disease; ORs: odds ratios; RCS: restricted cubic spline; SBP: systolic blood pressure; T2DM: type 2 diabetes mellitus; TC: total cholesterol; TG: triglycerides; UA: uric acid; UACR: urinary albumin to creatinine ratio

\section{Acknowledgements}

The authors are grateful to their colleagues and primary medical workers from Kangjian, Xujiahui, Huamu, Weifang, Gongyequ, Huangdu Community Health Care Centers, respectively, who kindly contributed to assisting the field data collection.

\section{Funding}

This study was supported by the Project of Shanghai Foundation for Senior Citizens (S15027), Construction Project of Key Discipline of Public Health in Shanghai (12GWZX1001), Science and Technology Project of Pudong New Area Commission of Health and Family Planning (PW2015C-25), Shanghai Municipal Commission of Health and Family Planning, Key Developing Disciplines (2015ZB0601).

Availability of data and materials

All data generated or analyzed during this study are included in this article

\section{Authors' contributions}

HY contributed to conception and design, acquisition of data, analysis and interpretation of data and drafting the article. DY contributed to conception and design and acquisition of data. JG contributed to conception and design and acquisition of data. YY contributed to conception and design and acquisition of data.MS contributed to conception and design and acquisition of data. YZ contributed to conception and design and acquisition of data. XD contributed to conception and design and acquisition of data.SZ substantially contributed to conception and design, revising the article critically for important intellectual content and final approval of the version to be published. XS substantially contributed to conception and design, revising the article critically for important intellectual content and final approval of the version to be published.

\section{Ethics approval and consent to participate}

The study protocol was approved by the Ethics Committee of Zhongshan Hospital of Fudan University (B2016-029). Written informed consent was obtained from all the participants.

\section{Consent for publication}

Written informed consent was obtained to publish from all the participants to report individual patient data. 


\section{Competing interests}

The authors declare that they have no competing interests.

\section{Publisher's Note}

Springer Nature remains neutral with regard to jurisdictional claims in published maps and institutional affiliations.

\section{Author details}

'Department of General Practice, Zhongshan Hospital of Fudan University, Shanghai, China. ${ }^{2}$ Department of General Practice, University of Melbourne, Carlton, Melbourne, VIC, Australia. ${ }^{3}$ Department of Nutrition, Zhongshan Hospital of Fudan University, Center of Clinical Epidemiology and Evidence-based Medicine, Fudan University, Shanghai, China. ${ }^{4}$ Department of Ophthalmology, Zhongshan Hospital of Fudan University, Shanghai, China. ${ }^{5}$ Department of General Practice, Shenzhen Longhua District Central Hospital, Shenzhen, Guangdong, China. ${ }^{6}$ Pudong Institute for Health Development, Shanghai, China.

\section{Received: 20 July 2018 Accepted: 8 January 2019}

\section{Published online: 18 January 2019}

\section{References}

1. Gheith O, Farouk N, Nampoory N, Halim MA, Al-Otaibi T. Diabetic kidney disease: world wide difference of prevalence and risk factors. J Nephropharmacol. 2016;5:49-56.

2. Rabkin R. Diabetic nephropathy. Clin Cornerstone. 2003:5:1-11.

3. Xiao Y, Sui BY, Zhao K. Epidemiological features of the end-stage renal disease and the application, cost and payment of dialysis care in China. Chinese Journal of Health Policy. 2011;05:29-33.

4. Yau JW, Rogers SL, Kawasaki R, Lamoureux EL, Kowalski JW, Bek T, et al. Global prevalence and major risk factors of diabetic retinopathy. Diabetes Care. 2012;35:556-64.

5. Mohamed Q, Gillies MC, Wong TY. Management of diabetic retinopathy: a systematic review. JAMA. 2007;298:902-16.

6. Rossing P. Prediction, progression and prevention of diabetic nephropathy. The Minkowski lecture 2005. Diabetologia. 2006;49:11-9.

7. Fong DS, Aiello L, Gardner TW, King GL, Blankenship G, Cavallerano JD, et al. Retinopathy in diabetes. Diabetes Care. 2004;27(Suppl 1):S84-7.

8. Fioretto P, Dodson PM, Ziegler D, Rosenson RS. Residual microvascular risk in diabetes: unmet needs and future directions. Nat Rev Endocrinol. 2010;6:19-25.

9. Ismail-Beigi F, Craven T, Banerji MA, Basile J, Calles J, Cohen RM, et al. Effect of intensive treatment of hyperglycaemia on microvascular outcomes in type 2 diabetes: an analysis of the ACCORD randomised trial. Lancet. 2010; 376:419-30.

10. Beulens JW, Patel A, Vingerling JR, Cruickshank JK, Hughes AD, Stanton A, et al. Effects of blood pressure lowering and intensive glucose control on the incidence and progression of retinopathy in patients with type 2 diabetes mellitus: a randomised controlled trial. Diabetologia. 2009:52:2027-36.

11. Dalrymple LS, Kaysen GA. The effect of lipoproteins on the development and progression of renal disease. Am J Nephrol. 2008:28:723-31.

12. Keen H, Lee ET, Russell D, Miki E, Bennett PH, Lu M. The appearance of retinopathy and progression to proliferative retinopathy: the $\mathrm{WHO}$ multinational study of vascular disease in diabetes. Diabetologia. 2001; 44(Suppl 2):S22-30.

13. Morton J, Zoungas S, Li Q, Patel AA, Chalmers J, Woodward M, et al. Low $\mathrm{HDL}$ cholesterol and the risk of diabetic nephropathy and retinopathy: results of the ADVANCE study. Diabetes Care. 2012;35:2201-6.

14. Sacks FM, Hermans MP, Fioretto P, Valensi P, Davis T, Horton E, et al. Association between plasma triglycerides and high-density lipoprotein cholesterol and microvascular kidney disease and retinopathy in type 2 diabetes mellitus: a global case-control study in 13 countries. Circulation. 2014;129:999-1008.

15. Zoppini G, Negri C, Stoico V, Casati S, Pichiri I, Bonora E. Triglyceride-highdensity lipoprotein cholesterol is associated with microvascular complications in type 2 diabetes mellitus. Metabolism. 2012;61:22-9.

16. Bose S, Bomback AS, Mehta NN, Chen SC, Li S, Whaley-Connell A, et al. Dysglycemia but not lipids is associated with abnormal urinary albumin excretion in diabetic kidney disease: a report from the kidney early evaluation program (KEEP). BMC Nephrol. 2012;13:104.

17. Sjolie AK, Stephenson J, Aldington S, Kohner E, Janka H, Stevens L, et al. Retinopathy and vision loss in insulin-dependent diabetes in Europe. The EURODIAB IDDM Complications Study. Ophthalmology. 1997;104:252-60.
18. Notice of the Ministry of Health on printing and distributing the Regulations of the National Basic Public Health Service (2011 Edition)[EB/ OL][http://www.gov.cn/zwgk/2011-05/24/content_1870181.htm].

19. KDOQI. KDIGO 2012 clinical practice guideline for the evaluation and management of chronic kidney disease. Kidney Int. 2013;Suppl 3:1-150.

20. Desquilbet $L$, Mariotti $F$. Dose-response analyses using restricted cubic spline functions in public health research. Stat Med. 2010;29:1037-57.

21. Stadler K, Goldberg IJ, Susztak K. The evolving understanding of the contribution of lipid metabolism to diabetic kidney disease. Curr Diab Rep. 2015;15:40.

22. Fakhrzadeh H, Ghaderpanahi M, Sharifi F, Badamchizade Z, Mirarefin M, Larijani B. Increased risk of chronic kidney disease in elderly with metabolic syndrome and high levels of C-reactive protein: Kahrizak elderly study. Kidney Blood Press Res. 2009;32:457-63.

23. Lee PH, Chang HY, Tung CW, Hsu YC, Lei CC, Chang HH, et al. Hypertriglyceridemia: an independent risk factor of chronic kidney disease in Taiwanese adults. Am J Med Sci. 2009;338:185-9.

24. Wang F, Ye P, Luo L, Xiao W, Wu H. Association of risk factors for cardiovascular disease and glomerular filtration rate: a community-based study of 4,925 adults in Beijing. Nephrol Dial Transplant. 2010:25:3924-31.

25. Yokoyama H, Sone H, Oishi M, Kawai K, Fukumoto Y, Kobayashi M. Prevalence of albuminuria and renal insufficiency and associated clinical factors in type 2 diabetes: the Japan diabetes clinical data management study (JDDM15). Nephrol Dial Transplant. 2009;24:1212-9.

26. Chen W, Chen W, Wang $\mathrm{H}$, Dong $\mathrm{X}$, Liu $\mathrm{Q}$, Mao $\mathrm{H}$, et al. Prevalence and risk factors associated with chronic kidney disease in an adult population from southern China. Nephrol Dial Transplant. 2009:24:1205-12.

27. Molitch ME, Rupp D, Carnethon M. Higher levels of HDL cholesterol are associated with a decreased likelihood of albuminuria in patients with longstanding type 1 diabetes. Diabetes Care. 2006;29:78-82.

28. Zoppini G, Targher G, Chonchol M, Perrone F, Lippi G, Muggeo M. Higher HDL cholesterol levels are associated with a lower incidence of chronic kidney disease in patients with type 2 diabetes. Nutr Metab Cardiovasc Dis. 2009;19:580-6.

29. Russo GT, De Cosmo S, Viazzi F, Pacilli A, Ceriello A, Genovese S, et al. Plasma triglycerides and $\mathrm{HDL}-\mathrm{C}$ levels predict the development of diabetic kidney disease in subjects with type 2 diabetes: the AMD annals initiative. Diabetes Care. 2016;39:2278-87.

30. Chang $\mathrm{YH}$, Chang DM, Lin KC, Hsieh CH, Lee YJ. High-density lipoprotein cholesterol and the risk of nephropathy in type 2 diabetic patients. Nutr Metab Cardiovasc Dis. 2013:23:751-7.

31. Yun KJ, Kim HJ, Kim MK, Kwon HS, Baek KH, Roh YJ, et al. Risk factors for the development and progression of diabetic kidney disease in patients with type 2 diabetes mellitus and advanced diabetic retinopathy. Diabetes Metab J. 2016;40:473-81

32. Kim SW, Jee JH, Kim HJ, Jin SM, Suh S, Bae JC, et al. Non-HDL-cholesterol/HDLcholesterol is a better predictor of metabolic syndrome and insulin resistance than apolipoprotein B/apolipoprotein A1. Int J Cardiol. 2013;168:2678-83.

33. Wen J, Zhong Y, Kuang C, Liao J, Chen Z, Yang Q. Lipoprotein ratios are better than conventional lipid parameters in predicting arterial stiffness in young men. J Clin Hypertens (Greenwich). 2017;19(8):771-6.

34. Zhu L, Lu Z, Zhu L, Ouyang X, Yang Y, He W, et al. Lipoprotein ratios are better than conventional lipid parameters in predicting coronary heart disease in Chinese Han people. Kardiol Pol. 2015;73:931-8.

35. Rema M, Srivastava BK, Anitha B, Deepa R, Mohan V. Association of serum lipids with diabetic retinopathy in urban south Indians--the Chennai urban rural epidemiology study (CURES) eye study-2. Diabet Med. 2006;23:1029-36.

36. Chew EY, Klein ML, Ferris FL 3rd, Remaley NA, Murphy RP, Chantry K, et al. Association of elevated serum lipid levels with retinal hard exudate in diabetic retinopathy. Early treatment diabetic retinopathy study (ETDRS) report 22. Arch Ophthalmol. 1996:114:1079-84.

37. Klein BE, Klein R, Moss SE. Is serum cholesterol associated with progression of diabetic retinopathy or macular edema in persons with younger-onset diabetes of long duration? Am J Ophthalmol. 1999;128:652-4.

38. van Dijk C, Berl T. Pathogenesis of diabetic nephropathy. Rev Endocr Metab Disord. 2004:5:237-48.

39. Knott RM, Forrester RM. Pathogenesis of diabetic retinopathy and cataract. In: Pickup JC, Williams G, editors. Textbook of Diabetes. Volume 2. 3rd ed. Oxford: Blackwell; 2003. p. 48.1-17.

40. Li J, Dong Y, Wu T, Tong N. Differences between Western and Asian type 2 diabetes patients in the incidence of vascular complications and mortality: a systematic review of randomized controlled trials on lowering blood glucose. J Diabetes. 2016:8:824-33. 